\title{
Abstracts
}

Cerebrovascular

Diseases

Cerebrovasc Dis 2007;24:483-494

DOI: $10.1159 / 000109749$

The abstracts are only available online, free of charge, under www.karger.com/doi/10.1159/000109749

\section{Third International Stroke Summit}

Wuhan, China, November 1-3, 2007

\section{Selected Abstracts}

Chairpersons

Xinfeng Liu, Nanjing, China

Markku Kaste, Helsinki, Finland

Shuming Zhang, Wuhan, China

Jiemei Zhang, Wuhan, China

Michael Chopp, Detroit, Mich., USA

Chengyan Li, Wuhan, China

Guohua Chen, Wuhan, China

Gelin $X u$, Nanjing, China 


\section{Cerebrovascular} Diseases

\section{Effect of Noninvasive Intranasal Delivery Nerve Growth Factor on Sarin-Induced Brain Damage}

\author{
X. Fan, G. Xu, W. Zhu, Y. Ma, X. Liu \\ Department of Neurology, Jinling Hospital, Nanjing \\ University School of Medicine, Nanjing, China
}

Background and Purpose: Sarin (O-isopropyl methylphosphonofluridate) is an organophosphorus that belongs to a group of highly toxic nerve agents. Sarin can induce severe and long-term brain damage, especially following status epilepticus induced by sarin. Presently the conventional therapy (atropine, pralidoxime et al.) is not better for this damage. Many patients were sequela of brain injured (e.g. palsy, remembrance disorder, cognitive handicap), even if they accepted these therapies as early as possible. Nerve growth factor (NGF) has the neuroprotective effect on brain injury induced by many etiological factors (e.g. ischemia, hypoxia, degeneration). But intravenous injection of NGF can not cross the blood brain barrier (BBB) efficiently because of its large molecular weight. And intracerebroventricular injection and intrathecal injection are invasive manipulations and have high risks of local infection and other serious complications. So these administrations are not useful in clinic. It was confirmed that NGF could cross the BBB into brain directly following intranasal (IN) administration. This study was aimed to investigate the effect of IN NGF on the brain damage of sarin-poisoned rats. Methods: Sprague-Dawley rats were treated with $80 \mu \mathrm{g} / \mathrm{kg}$ sarin following intramuscular injection (posterior limb muscle) and administered by peritoneal injection of atropine sulphate $(3 \mathrm{mg} / \mathrm{kg})$ and pralidoxime $(25 \mathrm{mg} / \mathrm{kg}) 1 \mathrm{~min}$ after intoxication. The rats were randomized to IN normal saline (NS) group and IN NGF group, which display typical toxic symptom 5-10 min after sarin injection, for example sialism, chew and convulsion. Each group had six rats. Normal control group was six normal rats. All rats were sacrificed 24 hours after sarin exposure. Damaged and residual healthy neurons were estimated and quantified on brain sections strained with hematoxylin-eosin (H\&E) mothod, and displayed neuronal nuclei antigen $(\mathrm{NeuN})$ with immunohistochemistry method. The regions of interest were piriform cortex, corpus striatum and hippocampus CA1. Results: As assessed by H\&E staining, a massive quantity of degenerated and necrotic neurons was observed in the piriform cortex, corpus striatum and hippocampus CA1 in rats from in IN NS group. Compared to the normal rats, NeuN-positive cells in IN NS group was significantly reduced by $39.44 \%, 46.26 \%$ and $54.10 \%$ respectively. But in IN NGF group the number of degenerated neurons was less than in IN NS group, and IN NGF significantly reduced the NeuN-positive cells loss. NeuN-positive cells in IN NGF group were reduced by $2.46 \%, 19.21 \%$ and $29.13 \%$ respectively in the piriform cortex, corpus striatum and hippocampus CA1. There was significantly different between IN NS group and IN NGF group $(p<0.05)$. Conclusions: Intranasal delivery of NGF could bypass the BBB and enter the central nerve system efficiently. IN NGF could improve neuropathology following sarin-exposure rats. NGF delivered by intranasal pathway, a noninvasive and cost-effective method, may be a potential treatment for sarin intoxication.

\section{Adjuvant Treatment with Atorvastatin Increases the Therapeutic Window for Thrombolysis after Embolic Focal Cerebral Ischemia by Activating the Phosphatidylinositol 3-Kinase/AKT-Signaling Pathway}

L. Zhang, Z.G. Zhang, X. Liu, A. Hozeska-Solgot, M. Chopp

Department of Neurology, Henry Ford Health System,

Detroit, Mich., USA

Background: Microvascular dysfunction post treatment of stroke with recombinant human tissue plasminogen activator (tPA) constrains the therapeutic window to $3 \mathrm{~h}$. Statins (3-Hydroxy-3methylglutaryl coenzyme A reductase inhibitors) have vascular beneficial effects well beyond reduction of cholesterol. In the present study, we investigated the neuroprotective effect of atorvastatin on thrombolysis and mechanisms underlying beneficial effects. Materials and Methods: Male Wistar rats were subjected to embolic middle cerebral artery occlusion (MCAo). Ischemic rats were treated with saline, atorvastatin $(20 \mathrm{mg} / \mathrm{kg})$ alone, tPA $(10 \mathrm{mg} / \mathrm{kg})$ alone, or combination of atorvastatin and tPA at $4 \mathrm{~h}$ after stroke. Results: Combination of atorvastatin and tPA significantly reduced ischemic lesion volume $(23.1 \pm 9.6 \%)$, whereas monotherapy with atorvastatin and IPA failed to reduce the lesion volume $(33.6 \pm 5.4 \%$ and $39 \pm 5.8 \%)$, when compared with saline treated rats $(35.6 \pm 10.2 \%)$, respectively. Western blot analysis revealed that treatment with atorvastatin alone and in combination treatment with tPA significantly increased Akt phosphorylation compared with treatment with saline and tPA alone. Inhibition of the PI3K/Akt pathway with wortmannin, a specific inhibitor of PI3K, completely abolished the reduction of lesion volume afforded by the combination of atorvastatin and tPA. Real time RT-PCR analysis of cerebral endothelial cells isolated by laser-capture microdissection from the ischemic boundary region showed that MCAo upregulated early growth response 1 (Egr-1) and vascular endothelial growth factor (VEGF) mRNA levels and tPA monotherapy further increased Egr-1 and VEGF mRNA levels. However, combination of atorvastatin and tPA significantly suppressed Egr-1 and VEGF mRNA levels, and downregulated expression of tissue factor, and matrix metalloproteinase 9 , which concomitantly reduced cerebral microvascular thrombosis and enhanced microvascular integrity. Conclusions: The combination treatment with atorvastatin and TPA exerts a neuroprotective effect when administered $4 \mathrm{~h}$ after stroke. The activation of the PI3K/Akt pathway by atorvastatin may suppress upregulation of Egr-1, and

\section{KARGER}

Fax +4161306 1234

E-Mail karger@karger.ch

www.karger.com
(C) 2007 S. Karger AG, Basel

1015-9770/07/0246-0483/\$23.50/0

Accessible online at:

www.karger.com/ced 
subsequently reduces the expression of multiple genes that promote thrombosis and vascular permeability, which increases the therapeutic window for thrombolysis after stroke.

\section{Intranasally Transporting NGF Gene into CNS by Recombinant HSV Vectors in Rats with Middle Cerebral Artery Infarction}

\author{
G. Xu, L. Bathala, X. Fan, X. Liu
}

Department of Neurology, Jinling Hospital, Nanjing University School of Medicine, Nanjing, Jiangsu Province, China

Objective: Several growth factors such as nerve growth factor (NGF) have been reported to be effective for neurogenesis and neuroprotecton after focal cerebral ischemia in animal model. But gene therapy for cerebrovascular disease in human still has many problems, of which lacking an effective delivery pathway to central nervous system (CNS) is a major hindrance for the clinical application of this promising strategy. This study was aimed to evaluate the feasibilty and efficacy of deliverying NGF gene with recombinant herpessimplex-virus vectors to CNS via intranasal olfactory pathway in rat. Method: Recombinant herpes-simplex-virus vector was constracted by deleting 6 viral genes, which included the coding sequence for an immediat early (IE) gene, ICP4. Focal cerebral ischemia was induced by middle cerebral artery occlusion (MCAO) with a nylon filament in Sprague-Dawley rats. MCAO rats were randomized into three groups: NGF, enhanced green fluorescent protein (eGFP) and control. Six hours after $\mathrm{MCAO}$, recombinant herpes-simplex-virus vectors carrying NGF gene or eGFP gene, a marker gene, or the void vectors were administrated intranasally. Bromodeoxyuridine (BrdU) was injected intraperitoneally twice daily on the fifth and sixth days for the purpose of neurogenesis measurement. On the seventh day and forteenth day after MCAO, the cerebral infarct volumes, neurogenesis, expression of NGF and eGFP were assessed. Neurological outcomes were serially assessed by the rotated test after MCAO. Results: Rats in NGF group scored higher in neurological function tests 7 and 14 days after MCAO compared with eGFP and control groups $(\mathrm{p}<0.05)$. Rats administrated with NGF gene has a trend of decreased infarct volume 14 days after MCAO compared with that of eGFP and control groups, but the differences did not reach the significant level $(p=0.10)$. Histological detections revealed that expression of NGF in hippocampus and temporal lobe were significantly enhenced 7 and 14 days after MCAO in NGF group in contrast to eGFP and control groups $(p<0.05)$. Green fluorescence was also detected in hippocampus and temporal lobe of rats administrated with eGFP gene. The BrdU-positive cells in subventricular zone and hippocampus was significantly increased in NGF gene treated rats than eGFP treated and control rats $(p<0.05)$. Conclusions: Recombinant herpessimplex-virus vectors administrated via intranasal olfactory pathway can efficiently delivering NGF gene into CNS and expressing. NGF gene therapy is efficacious in treating focal cerebral ischemia in rats.

Third International Stroke Summit

\section{The Preventive Effects of Minocyline in Hemorrhagic Transformation after Thrombolytic Therapy of Acute Brain Infarction in Rats}

\author{
Y. Zhao, J. Tang
}

Department of Neurology, the First People's Hospital of Shanghai Jiaotong University, Shanghai, China

Objective: To construct a rat model which can be used for study of thrombolytic therapy 6 hours after brain infarction onset? To study the preventive effects of minocyline in hemorrhage transformation after thrombolytic therapy of acute brain infarction in rats. Methods: Male Spraque-Dawley rats were divided into A, B, C and D groups. The diameter of the clots used in group A was $0.3 \mathrm{~mm}$ while which of the others was $0.4 \mathrm{~mm}$. After 3 and 6 hours of embolization, neurological deficits of rats in group A and B were evaluated, then rats were killed and the thromboembolic conditions in MCA were inspected. After that, coronary sections underwent TTC dyeing to calculate the percentage of infarct brain in the hemisphere by Photoshop. Rats in group $\mathrm{C}$ and $\mathrm{D}$ received urokinase 6 hours after infarction. Before that, rats in group $\mathrm{C}$ and $\mathrm{D}$ were given minocycline and saline respectively. Neurological function scores were determined at 3, 6, 24 hours after brain infarction, and then rats were killed with a lethal overdose of halothane and transcardially perfused to remove all intravascular blood. Hemispheric brains were homogenized and ultrasonicated on ice. After centrifugation, the supernatant was collected. Cerebral hemorrhage was quantified with a spectrophotometer assay. Results: The percentage of infracted brain in the hemisphere and neurological function scores of group A were higher than group B $(p<0.05)$. All rats in group A were obstructed fully in the conjoining position of MCA and ICA, but only 1 rat in group B was obstructed at the same place. Hemorrhage volumes of rats in group $\mathrm{C}$ were larger than which in group D (13.34 \pm 4.75 vs. $6.89 \pm 4.97, \mathrm{p}<0.05)$. Neurological scores and mortalities were not different significantly between group $\mathrm{C}$ and D ( $>$ > 0.05). Conclusions: MCA of male SD rats can be occluded fully with clot which diameter is $0.4 \mathrm{~mm}$ for at least 6 hours. It can be used for thrombolytic therapy 6 hours after brain infarction. Minocycline can reduce the hemorrhage volumes after thrombolytic therapy in thromboembolic rat models.

\section{G-CSF Activating for Auto-Protection Mechanism of Brain at Persistent Hypo-Blood-Stream State}

\author{
X. Shu, H. Zhou, Y. Li, L. Zhang, O. Chen, L. Liu \\ Department of Pathology, School of Medicine and Life \\ Science, Jianghan University, China
}

Aim: Using G-CSF excite auto-protection mechanism of brain at persistent hypo-bloodstream state, to research the reactive and morphological changes of astrocyte in hippocampus CAI domain. Materials and Methods: The 2 vessel occlusion (2VO) model was established. 2VO group and control group (sham operated group) all had 15 rats and were gave rhG-CSF all groups three days continuously and use isodose physiologic saline as control $20 \mathrm{w}$ after operation. 
Ten rats from $2 \mathrm{VO}$ group and control group each were sacrificed 1d after gave rhG-CSF and physiologic saline. Another rats were sacrificed $5 \mathrm{~d}$ after gave rhG-CSF and physiologic saline. Bone marrow cell was detected by CD34. Nerve stem cell was detected by Nestin and $\beta$-tublin. Cell proliferation was detected by PCNA index number. Apoptosis was detected by Hoechest33258. Astrocyte was detected by the immunohistochemical staining of GFAP. Result: When gave rhG-CSF, CD34 positive cell could be observed rat's brain. This was the proof that bone marrow cell was mobilized and moved to brain. When gave rhG-CSF continuously, Nestin positive nerve stem cell could be observed in different place of rat's brain, and this appearance was the proof that bone marrow cell which was mobilized and moved to brain had transformed or mobilized to nerve stem cell. Numbers of PCNA positive cells of $2 \mathrm{VO}$ and rhG-CSF treatment group in hippocampus $\mathrm{CAI}$ domain was evidence more than $2 \mathrm{VO}$ and normal sodium treatment group. Numbers of $\beta$-tublin positive cells of $2 \mathrm{VO}$ and rhG-CSF treatment group was evidence difference of control group and normal sodium treatment group. Numbers of apoptosis cells in hippocampus CAI domain was evidence less than normal sodium treatment group. Numbers of astrocyte was evidence increased and the length of astrocyte ecphyma was evidence shorten that was different with control group. Conclusion: Using G-CSF excite auto-protection mechanism of brain at persistent hypo-bloodstream state, changes of number of astrocyte and length of astrocyte ecphyma proved astrocyte participate repairing proceed of nerve cell.

\section{The Effect of Leukotrienes $C_{4}$ on Human Umbilical Vein Endothelial Cells}

\author{
W. Zhu, G. Zhou, M. Ma, X. Fan, G. Xu, X. Liu \\ Department of Neurology, Jinling Hospital, Nanjing \\ University School of Medicine, Nanjing, China
}

Objective: To investigate the effect of leukotrienes $\mathrm{C}_{4}\left(\mathrm{LTC}_{4}\right)$, metabolites of arachidonic acid on human umbilical vein endothelial cells (HUVECs). Methods: Isolated and cultured HUVECs were used as experimental model. HUVECs were induced by $\mathrm{LTC}_{4}$ at different concentrations. It can be divided with 5 groups: control group, $10^{-10} \mathrm{~mol} / 1 \mathrm{LTC}_{4}$ group, $10^{-8} \mathrm{~mol} / 1 \mathrm{LTC}_{4}$ group, $10^{-6} \mathrm{~mol} / 1 \mathrm{LTC}_{4}$ group and leukotriene receptor antagonist BAY u9773 group $\left(10^{-6} \mathrm{~mol} / 1 \mathrm{LTC}_{4}+1 \mu \mathrm{mol} / 1 \mathrm{BAY} \mathrm{u} 9773\right)$. Each group had 4 samples. 24 hours after incubation, lactate dehydrogenase (LDH), malonaldehyde (MDA) were detected with chromatometry and cell apoptosis was detected with flow cytometry. Results: There was no difference in $\mathrm{LDH}, \mathrm{MDA}$ and cell apoptosis between $10^{-10} \mathrm{~mol} / 1 \mathrm{LTC}_{4}$ group and control group. $\mathrm{LDH}, \mathrm{MDA}$ and cell apoptosis in $10^{-8} \mathrm{~mol} / 1 \mathrm{LTC}_{4}$ group and $10^{-6} \mathrm{~mol} / 1 \mathrm{LTC}_{4}$ group were higher than those in control group. $\mathrm{LDH}, \mathrm{MDA}$ and cell apoptosis in BAY u9773 group were lower than those in $10^{-6} \mathrm{~mol} / 1 \mathrm{LTC}_{4}$ group. Conclusion: $\mathrm{LTC}_{4}$ above $10^{-8} \mathrm{~mol} / 1$ induces HUVECs injury and cell apoptosis. Leukotrienes receptor antagonist BAY u9773 can relieve the injury in HUVECs induced by $\mathrm{LTC}_{4}$.

\section{Optimal Dosages of Intravenous and Intranasal Cerebrolysin in Treatment of Stroke}

\author{
X. Liu, G. Xu, R. Zhang, W. Zhu, L. Bathala \\ Department of Neurology, Jinling Hospital, Nanjing, China
}

Objective: To determine the optimal dosages of intravenous (IV), and intranasal (IN) Cerebrolysin for the treatment of stroke. Methods: Sprague-Dawley 52 rats were randomized into four groups as I.V. vehicle group, $\mathrm{n}=10, \mathrm{I}$.V. low $(1.0 \mathrm{ml} / \mathrm{kg}), \mathrm{n}=8, \mathrm{I}$.V. middle $(2.5 \mathrm{ml} / \mathrm{kg}), \mathrm{n}=8$, and I.V. high Cerebrolysin-treated group $(5.0 \mathrm{ml} / \mathrm{kg}), \mathrm{n}=8$, and two groups as I.N. $2.25 \mathrm{mg}$ Cerebrolysin, $\mathrm{n}=9$ and I.N. vehicle groups, $\mathrm{n}=9$. All were anesthetized and focal cerebral ischemia was induced by MCAO method. I.V. Cerebrolysin was administered at $0,2,24$, and 48 hours as a bolus injection through the tail vein. For I.N. delivery, $56 \mu 1(40.05 \mathrm{mg} / \mathrm{ml}$ Cerebrolysin or saline vehicle) solutions were administered. Behavioral neurological deficits were assessed prior to and at three hour interval after the onset of MCAO. Rats were euthanized at $72 \mathrm{~h}$, the brain sections were stained and an image analysis system was used to calculate the infarct volume. Results: Infarct volume after MCAO significantly reduced in rats were received the $2.5 \mathrm{ml} / \mathrm{kg}$ dose of IV Cerebrolysin $(\mathrm{p}<0.01)$. A dose of $2.5 \mathrm{ml} / \mathrm{kg}$ and $5.0 \mathrm{ml} / \mathrm{kg} \mathrm{I.V}$. Cerebrolysin group significantly improved performance on the postural reflex, the beam balance and the adhesive tape tests. No consistent differences $(p>0.05)$ in behavioral performance after MCAO were seen between the I.V. vehicle group, I.V. low $(1.0 \mathrm{ml} / \mathrm{kg})$, and I.V. high Cerebrolysin-treated group $(5.0 \mathrm{ml} / \mathrm{kg})$. Intranasal delivered Cerebrolysin significantly decreased infarct volume after MCAO $(p<0.01)$. No beneficial behavioral effect was identified at this one dose. There were no difference between the I.N. vehicle and I.N. Cerebrolysin-treated group for performance in postural reflex, beam balance test, or adhesive tape test. Conclusions: Both IV and IN Cerebrolysin reduce infarct volume after MCAO. The middle dose of IV Cerebrolysin $(2.5 \mathrm{ml} / \mathrm{kg})$ was most effective at reducing infarct volume and behavioral deficits. Intranasal delivered Cerebrolysin significantly reduced infarct volume, but not behavioral deficits after MCAO at a dose of $2.25 \mathrm{mg}$ suggesting this is not the optimal intranasal dose.

\section{Relationship between Polymorphism of Angiotensin Converting Enzyme Gene and Risk for Stroke in Elderly}

W. Zhao, H. Ji, W. Zhu

Department of Neurology, Nanjing General Hospital of Nanjing Military Command, Nanjing, Jiangsu, China

Objective: To investigate the relationship between angiotensin converting enzyme (ACE) gene polymorphism and risk for stroke in elderly. Methods: Frequencies of ACE gene type and allele were sequenced in 186 stroke patients and 75 normal controls by polymerase chain reaction restriction fragment length polymorphism (PCR-RFLP) technique. The stroke patients included 126 patients with cerebral infarction and 60 patients with cerebral hemorrhage. 
The PCR determined sequence located in the 16 intron with $287 \mathrm{bp}$. Results: Frequency of D allele was $41.9 \%$ in stroke patients and $31.3 \%$ in the controls $(p<0.05)$; Frequency of DD genotype was $21.5 \%$ in stroke patients and $9.3 \%$ in controls. The frequencies of DD genotype and $\mathrm{D}$ allele in cerebral hemorrhage and cerebral infarction groups was significantly higher than that in control group $(\mathrm{p}<0.05)$. Conclusions: The DD gene type of $\mathrm{ACE}$ and $\mathrm{D}$ allele may be a risk factor for stroke in elderly.

\section{The Histopathological Changes of Rat's CCH}

\author{
H. Zhou, X. Shu, L. Zhang, Y. Li, C. Wang, L. Liu
}

Department of Pathology, School of Medicine and Life Science, Jianghan University, China

Aim: Detecting the morphological changes cortical layer, callosities and hippocampus CAI domain and their relationships. Materials and Methods: The 2 vessel occlusion (2VO) model was established. 2VO group and control group (sham operated group) all had 19 rats and were sacrificed $28 \mathrm{~d}, 60 \mathrm{~d}, 28 \mathrm{w}$ and $50 \mathrm{w}$ after operation. All tissues were routine paraffin imbedded; hematoxylin and eosin stained and observed morphological change of horizontal cell in cortical layer, callosities and hippocampus CAI domain in microscope. The Immunofluorescence staining of Hoechest33258 was performed according to the directions. And apoptosis was observed in cortical layer, callosities and hippocampus CAI domain. Using image analysis technique collects the image of those domains, counting the number of nerve cell, glia cell and apoptosis cell per $34784.6 \mu \mathrm{m}^{2}$. ALL data were analyzed by the analysis of variance, $t$ test, $F$ test and coefficient correlation analysis. A $p$ value $<0.05$ was considered to indicate statistical significance. Result: The numbers of nerve cell of cerebral cortex in $28 \mathrm{~d}, 28 \mathrm{w}$ and $50 \mathrm{w}$ after $2 \mathrm{VO}$ operation were obviously less than control group. The numbers of apoptosis cell of cerebral cortex in $28 \mathrm{~d}, 60 \mathrm{~d}$ and $28 \mathrm{w}$ after $2 \mathrm{VO}$ operation were obviously more than control group and in $50 \mathrm{w}$ after $2 \mathrm{VO}$ operation were obviously less than control group. The numbers of pyramidal cell of hippocampus CAI domain in $28 \mathrm{~d}, 60 \mathrm{~d}, 28 \mathrm{w}$ and $50 \mathrm{w}$ after $2 \mathrm{VO}$ operation were obviously less than control group. The numbers of apoptosis cell of cerebral cortex in $28 \mathrm{~d}, 60 \mathrm{~d}, 28 \mathrm{w}$ and $50 \mathrm{w}$ after $2 \mathrm{VO}$ operation were obviously less than control group. The numbers of glial cell of callositas in $28 \mathrm{~d}, 60 \mathrm{~d}, 28 \mathrm{w}$ and $50 \mathrm{w}$ after $2 \mathrm{VO}$ operation were obviously less than control group. The nerve cell of cerebral cortex appeared conspicuously neuronophagia and hippocampus CAI domain appeared gumming; gummosis in $50 \mathrm{w}$ after $2 \mathrm{VO}$ operation. Small vessels obviously expanded. Conclusion: Longer time of $\mathrm{CCH}$, the number of nerve cell of cerebral cortex was less. The number of nerve cell of cerebral cortex was related of apoptosis in earlier period and was related of nerve cell degeneration and necrosis in advanced stage. The numbers of pyramidal cell of hippocampus CAI domain was decreased gradually and was related of apoptosis.

\section{Awareness of Symptoms and Risk Factors of Stroke among Stroke Patients in South West India}

\author{
L. Bathala, S.N. Rao, N. Thambyrajah, V. Guddattu \\ Department of Neurology, Kasturba Medical College, \\ Manipal, India
}

Objective: To determine the awareness of symptoms and risk factors of stroke among stroke patients in Southern India. Methods: This study design is a cross sectional hospital-based study. Subjects were patients with cerebrovascular accident admitted to the department of neurology Kasturba hospital Manipal, from January to May 2007. Trained neurology residents, interviewed the subjects using a modified, pretested, open-ended questionnaire obtained from Dr Pancioli Arthur, Department of Emergency Medicine, University of Cincinnati, Ohio. Symptoms and risk factors of stroke were those specified by the National institute of Neurological disorders and Stroke (NINDS). In case patients cannot be interviewed due to deficits their close relatives took part in the study. Results: A total of 50 (70\% males and $54 \%$ above 40 years of age) subjects participated in this study. Only $26 \%$ identified brain as the organ of stroke. $52 \%$ knew at least one risk factor and $56 \%$ one symptom of stroke. In multivariate analysis higher income correlated with better awareness of stroke symptoms and risk factors ( $\mathrm{p}<0.031$ and $<0.027$ respectively). Among the risk factors subjects knew hypertension $40 \%$, diabetes $22 \%$, smoking $18 \%$, heart disease $8 \%$ and elevated cholesterol $2 \%$ could cause stroke. Ninety four percent were not aware of the time window and thrombolytic therapy for stroke. Sixty percent preferred doctors over other sources to obtain information regarding stroke. Conclusions: This hospitalbased survey reveals a suboptimal awareness regarding stroke among patients. As majority of patients feel doctors are reliable over other sources to obtain information, considerable effort is needed from health care providers to increase public awareness regarding risk factors, symptoms and treatment of stroke.

\section{Functional MRI of Rat's CCH in Hippocampus CA1 Domain}

L. Zhang, H. Zhou, Y. Li, Y. Xiang, X. Shu, L. Liu

Department of Pathology, School of Medicine and Life Science, Jianghan University, China

Aim: Using ${ }^{1} \mathrm{H}$ Magnetic Resonance Spectrum (MRS) in vivo, detected the biochemics changes in brain at $\mathrm{CCH}$ state, to perform the nerve injury mechanism of hippocampus when cerebral blood flow decreased. Materials and Methods: The 2 vessel occlusion (2VO) model was established. $2 \mathrm{VO}$ group and control group (sham operated group) all had 3 rats and were detected by MRS method to detected ${ }^{1} \mathrm{H}$ spectra of hippocampus domain $28 \mathrm{~d}$ after operation. ALL data were analyzed by $t$ test. A p value $<0.05$ was considered to indicate statistical significance. Result: The relative magnitude of metabolin $\mathrm{NAA} /(\mathrm{Cho}+\mathrm{Cr})$ in hippocampus CAI domain was $0.61 \pm 0.04$ in the $2 \mathrm{VO}$ group $50 \mathrm{w}$ after operation. The relative magnitude of metabolin $\mathrm{NAA} /(\mathrm{Cho}+\mathrm{Cr})$ in hippocampus CAI domain was $0.61 \pm 0.04$ in the control group $50 \mathrm{w}$ after operation. There was significant different 
between two of them $(\mathrm{p}=0.04)$. The lactic acid signal did not appear in control group. There was one rat appeared lactic acid signal in the 2VO group 50w after operation. Conclusion: The result of MRI show content of NAA/(Cho $+\mathrm{Cr})$ decreased in hippocampus CAI domain, proof the lesion of pyramidal cell.

\section{Feasibility of Treating Intracranial Artery Stenosis with Stenting}

X. Liu, G. Xu, Q. Yin, R. Zhang

Department of Neurology, Jinling Hospital, Nanjing University School of Medicine, Nanjing, China

Objective: This study was aimed to analyzing the short-term angiographic follow-up results and midterm clinical outcomes in patients with symptomatic intracranial atherosclerotic lesions treated with stent placement. Methods: Thirty-nine patients with ischemic symptoms referable to stenotic intracranial atherosclerotic arteries, with greater than $70 \%$ stenosis, underwent elective surgery in which a primary stent was placed. All patients underwent pretreatment with combination oral antiplatelet (clopidogrel and aspirin) therapy for at least 3 days. Long-term (6-month or longer) combination oral antiplatelet (clopidogrel and aspirin) therapy after procedures were placed. The procedure involved selecting stents of the same size as the diameter of the target vessel and slowly inflating the balloon to its nominal pressure. Short-term angiography and midterm clinical follow-up data were obtained. Results: The stents were successfully implanted in all patients without any periprocedure complications. The mean preoperative stenosis rate of $79.3 \%$ was decreased to $12.7 \%$ after the stent implantation. Twenty-one patients who underwent follow-up angiography at a mean of 10.4 months (range 16-20 months) had no significant changes in luminal diameter compared with the immediate postoperative luminal diameter $(12.7 \%$ to $19.4 \%$, $\mathrm{p}<0.05$ ). Luminal narrowing increased more than $30 \%$ in two cases in whom no marked clinical symptoms manifested. No patient suffered new ischemic symptoms during a mean clinical follow-up period of 17.4 months (range 6-23 months). Conclusions: Elective stent implantation can provide good angiographic and clinical midterm outcomes in patients with symptomatic intracranial atherosclerotic stenosis, and the procedure is associated with a high degree of technical success. Reassessment of these promising results is needed in a larger population and in a randomized prospective comparison study.

\section{Study of Neurological Complications after Coronary Artery Bypass Grafting}

Q. Bi, Q. Li, Z. Zhang, Z. Zhang, X. Ma, C. Gu

Department of Neurology, Beijing Anzhen Hospital, Capital Medical University, Beijing, China

Objective: To prospectively investigate neurological complications (NC) after off-pump coronary artery bypass grafting (OPCABG), and to study sort of $\mathrm{NC}$, mobility, risk factors, prevention and treatment.
Materials and Methods: A total of 55 consecutive patients with OPCABG were evaluated from March to June 2006 at Department of Cardiosurgery in Beijing Anzhen Hospital with questionnaire to collect risk factors of NC before operation; Nervous system physical examination, NIHSS score evaluation, cognitive tests, image (MRI, MRA and f-MRI), anxious and depression scale were performed before and after OPCABG, and identify the $\mathrm{NC}$ and sorts after surgery; According to with or without $\mathrm{NC}$ after operation, all patients were divided into two groups, and univariate analysis was used between tow groups, $\mathrm{p}<0.05$ was considered statistically significant different. Result: (1) 2 cases of ischemic stroke developed after surgery $(3.64 \%, 2 / 55)$, one concerning posterior cerebral artery (PCA), another involving middle cerebral artery(MCA) and no death, however, the risk factors not statistically different between the patients with or without NC group ( $\mathrm{p}>0.05$ ); (2) for all patients, cognitive evaluation was normal and unchanged before and after surgery $(\mathrm{p}>0.05)$, including Mini-Mental State Examination (MMSE), Clinical dementia rating (CDR) and Global deterioration scale (GDS); (3) except 2 cases of stroke, the others normal and unchanged on MRI, MRA, and f-MRI ( $p>0.05)$ before and after surgery; (4) 7 patients with slight anxiety and one with slight depression before operation, recovered completely after surgery without treatment, but for all patients, both Self-rating anxiety scale (SAS) and Self-rating depression scale (SDS) uncha $4.8 \pm 0.8$ for NGF group and $4.7 \pm 0.9$ nged before and after surgery $(p>0.05)$. Conclusions: 2 cases of ischemic stroke after OPCABG $(3.64 \%, 2 / 55)$ and no death; but no change obviously about cognitive function, images, anxiety, depression before and after surgery $(\mathrm{p}>0.05)$. OPCABG was relatively safety for nerve system, but need more clinical data to explore.

\section{Retrospective Analysis of Risk Factors Associated with Blood Lipid Levels for Patients with Cerebral Infarction in Wuhan District}

G. Chen, X. Qiu, B. Liu, X. Wang, B. An

Department of Neurology, Integrated Traditional Chinese and Western Medicine Hospital of Tongji Medical College of Huazhong University of Science and Technology,

Wuhan, China

Objective: To investigate the relation between blood lipid and risk factors for the patients with cerebral infarction in Wuhan District. The risk factors include hypertension, diabetes, smoking, drinking, heart disease, genetic factors and age. Method: Data of 1,403 patients admitted to our Department in Wuhan from December 2002 to February 2007 were analyzed. They were diagnosed as cerebral infarction by brain CT and MRI. We retrospectively observed the relation between blood lipid and risk factors for the patients by linear regression analysis (SAS 8.2). Results: Triglyceride, total cholesterol, low-density lipoprotein were compared with hypertension, diabetes, smoking, drinking, heart disease, genetic factors and age respectively. $\mathrm{r}$ and $\mathrm{p}$ values between Triglyceride and above risk factors were: -0.0169 (0.5969), 0.07487 (0.0071), -0.01637 (0.5592), 0.00449 (0.8729), -0.0699 (0.0006), -0.09707 (0.117), 0.04376 (0.0238); $\mathrm{r}$ and $\mathrm{p}$ values between total cholesterol and above risk factors were: -0.02117 (0.5075), $0.09883(0.0004),-0.08802$ (0.0017), 
0.01787 (0.017), - 0.06729 (0.1747), 0.03179 (0.255), -0.04201 (0.5239); Between low-density lipid protein and above risk factors, $\mathrm{r}$ and $\mathrm{p}$ values were shown as below: -0.01389 (0.6638), 0.06585 (0.0179), -0.03492 (0.2127), 0.05638 (0.0442), -0.04974 (0.3341), -0.02726 (0.1996), $0.03582(0.108)$. Conclusion: In patients with cerebral infarction in Wuhan district, triglyceride was closely related to diabetes, heart disease and age. Total cholesterol was correlated to diabetes, smoking and drinking. Low-density lipoprotein was correlated to diabetes, smoking and genetic factors. Triglyceride was positively correlated to age. Diabetes was positively correlated to triglyceride, total cholesterol and low-density lipoprotein. Drinking was positively correlated to total cholesterol and low-density lipoprotein. Therefore, it was helpful to obtain optimal blood lipid level by controlling diabetes and drinking of patients with cerebral infarction in Wuhan district.

\section{Perimesencephalic Nonaneurysmal Subarachnoid Hemorrhage: Clinical Analysis of 3 Cases}

Y. Cui, Y. Cai, J. Cai

Department of Neurology, No 306 Hospital of PLA, Beijing, China

Objective: To investigate the feature of clinic and imaging, diagnosis, treatment and prognosis of Perimesencephalic nonaneurysmal subarachnoid hemorrhage. Methods: We presented a retrospective analysis for the clinical and radiological manifestation, treatment and prognosis of three cases. Results: All patients were conscious, who's Hunt-Hess scale were Grade I to II. All the CT scan revealed SAH located perimesencephalic cistern. Cerebral Angiography failed to show any positive finding. All patients treated with medical therapy and hadn't any complications such as rebreeding, cerebrovascular vasospasm and hydrocephalus. All patients recovered completely. Conclusion: PNSH is a distinct clinical entity with few manifestations, good prognosis and low rate of severe complications. It is important that the correct diagnosis of PNSH to avoid unnecessary surgical exploration and repeated angiograph.

\section{Carotid Stenting with Embolic Protection: Single-Center Experience}

Q. Yin, G. Xu, X. Liu, R. Zhang

Department of Neurology, Jinling Hospital, Nanjing University School of Medicine, Nanjing, China

Purpose: This study aimed to evaluate the efficacy and safety of carotid artery stent implantation with embolic protection. Materials and Methods: During a 3-year period (January 2003 to December 2005), 107 patients underwent carotid artery stent implantation with an embolic protection device. There were 77 male (mean age, 64.1 years; age range from 48 to 76 years) and 30 female (mean age, 63.5 years; age range from 56 to 82 years). Eighty-two patients were symptomatic and 25 patients were asymptomatic. All patients were at normal risk for carotid endarterectomy. Results: The procedure was technically successful in all 106 patients at the first attempt. The procedure was stopped in one patient due to uncontrolled restlessness. In one case, the carotid stent was implanted successfully one week late on the second attempt under the general anesthesia with propofol. During the procedure, 8 patients $(7.5 \%)$ experienced severe bradycardia or sustained hypotension. One patient $(0.93 \%)$ developed a minor stroke which recovered in 4 days. The mean follow-up was 14 months (range, 12-26 months). Two patients died, with one from phenomena and the other from heart failure. No stent occlusion was observed. During the late follow-up period $(>30$ days), one patients $(0.93 \%)$ had minor strokes, two patients $(1.86 \%)$ had transient ischemic attacks. Conclusion: Carotid artery stenting with cerebral protection by using the specific devices compares favorably to the previously reported surgical results.

\section{The Analysis of Risk Factors of Carotid Plaque in Patients with Cerebral Infarction in Wuhan}

\author{
X. Qiu, G. Chen, B. An, B. Liu, X. Wang
}

Department of Neurology, Integrated Traditional Chinese and Western Medicine Hospital of Tongji, Medical College of Huazhong University of Science and Technology, Wuhan, Hubei, China

Objective: To analyze the risk factors related to carotid plaques in patients with cerebral infarction. Methods: 572 unselected patients with cerebral infarction diagnosed by CT or MRI scans from December 2002 to February 2007 were studied. All of them had performed color Doppler inspections. Single plaque at carotid artery had been found in 132 patients, multiple plaques could be detected in carotid arteries of 169 patients, and 271 patients were normal. Risk factors probably related with carotid plaques, such as hypertension, diabetes, cholesterol, triglyceride, low-density lipoprotein, hematocrit, genetic factor, drinking, profession, gender were selected for study. The ages were assessed at a ten-year interval. Risk factors screening have been done by logistic regression analysis. Result: There were significant differences in the incidence of plaques in different aged groups $(\mathrm{F}=11.54$, $\mathrm{p}<0.0001)$. The hematocrit in patients with multiple plaques were significantly different from those of the other two groups $(\mathrm{F}=7.23$, $\mathrm{p}=0.0008$ ), while there was no obvious difference could be found between the single plaque and normal group. There was a distinct difference in smoking factors between the normal and the multiple plaques group $\left(X^{2}=6.1259, p=0.0133\right)$, whereas we could not find clear difference neither between single plaque and the normal group $\left(\mathrm{X}^{2}=2.8981, \mathrm{p}=0.0887\right)$, nor between the single plaque and the multiple plaques group $\left(\mathrm{X}^{2}=2.2817, \mathrm{p}=0.5956\right)$. The other factors such as hypertension $(p=0.5751)$, diabetes $(p=0.9145)$, cholesterol $(\mathrm{p}=0.2546)$, triglyceride $(\mathrm{p}=0.4631)$, low-density lipoprotein $(\mathrm{p}=0.5619)$, genetic factor $(\mathrm{p}=0.5292)$, drinking $(\mathrm{p}=0.974)$, profession $(p=0.0823)$, gender $(p=0.3096)$, had no significant relationship with the incidence of plaque. Logistic regression model analyzer showed the two factors, smoking and age, had statistical influence to the incidence of plaque. The OR of age and smoking for carotid plaques were $1.528(p=0.001), 1.548(p=0.0372)$ respectively. Conclusion: Hematocrit, age and smoking were the potential risk factors for carotid plaque formation in the patients of cerebral infarction in Wuhan. Age 
and smoking were the possible independent risk factors. The incidence of carotid plaques increased in recent 10 years. The incidence of carotid plaques of smoking patients was 1.548 times higher than that of non-smoking patients.

\section{Prominent Laterality of the Posterior Cerebral Artery Displayed on 3D-TOF-MRA in Middle Cerebral Artery Stem Occlusion: Prediction Values for Infarction Extent and Clinical outcomes}

\author{
H. Chen, S. Ge, G. Xu, X. Liu, R. Zhang, O. Yin \\ Department of Neurology, Jinling Hospital, Nanjing \\ University School of Medicine, Nanjing, China
}

Background and Purpose: Prominent laterality of the posterior cerebral artery (PLPCA) has often been observed at 3D-TOF-MRA in patients with middle cerebral artery (MCA) stem occlusion, and it may indicate distinguished collateral flow from PCA to MCA area. The purpose of this study is to evaluate the effect of the PLPCA at 3D-TOFMRA in patients with MCA stem occlusion for infarction extent and clinical outcomes of stroke. Methods: We retrospectively reviewed neuron-imaging results and clinical characters at 27 patients (20 male, 7 female; age range $42-86$ years, mean age $61.6 \pm 10.5$ years) who performed 3D-TOF-MRA within 24 hours from onset and demonstrated middle cerebral artery stem occlusion. According to the results of MRA, we divided these patients into PLPCA group and nonPLPCA group. On the basis of display at brain CT or/and MRI after one week from stroke onset, we scored the final extent of infarction. We tested patient's NIHSS within 24 hours of stroke, and made mRS in follow-up. Then, two group's infarction extent, NIHSS and mRS were compared. Results: The PLPCA group's infarction extent score, NIHSS and mRS was lower than non-PLPCA group's (infarction extent score: $1.38 \pm 1.04$ vs. $2.50 \pm 1.35, \mathrm{p}=0.024$; NIHSS on admission: $7.23 \pm 4.66$ vs. $11.50 \pm 5.76, p=0.045 ; \mathrm{mRS}$ in follow-up: $1.62 \pm 1.19$ vs. $2.93 \pm 1.44, p=0.017)$. Conclusions: Patient with MCA stem occlusion, PLPCA was displayed at 3D-TOF-MRA, may have smaller infarction and better clinical prognosis.

\section{Stenting of Atherosclerotic Intracranial Stenosis: Effect and Safety}

F. Zhu, X. Fan

Department of Neurology, Jinling Hospital, Medical

College of Nanjing University, Nanjing, China

Objective: To observe the feasibility and safety of intracranial arterial stenting for treating the patients with symptomatic Intracranial arterial stenosis. Methods: 33 patients who were diagnosed as intracranial stenosis by DSA from February 2004 to July 2007. Among them, 15 cases were the intracranial stenosis of arteria carotis, 12 cases were the intracranial stenosis of vertebral artery and basilar artery. 6 cases were the stenosis of middle cerebral artery. The degree of stenosis was $50 \% \sim 70 \%$ in 12 patients, $71 \% \sim 95 \%$ in 21 patients. 33 patients were Anesed and stents were placed. Results: The degree of stenosis was $71.5 \pm 18.5 \%$ in 33 cases, After stent the plaques were disappear in 28 cases, the degree of stenosis was decreased to $12.5 \pm 8.2 \%$ in 5 cases. The clinical symptoms of 32 patients were relieved, one patient died of intracranial haemorrhage, one patient suffered from haematoma in puncted arteria cruralis. 8 patients were rechecked in 6-26 months, 7 patients had no restenosis, 1 patient suffered from restenosis (25\%). Conclusions: Stenting of atherosclerotic intracranial Stenosis is a safe and effective method for treatment symptomatic intracranial Stenosis. intracranial stent lower the risk of arterothrombotic cerebral infarction, TIA and lacunar infarction.

\section{Factors Influencing the Length of Stay (LOS) of Cerebral Infarction Patients in Wuhan}

\author{
G. Chen, X. Qiu, X. Wang, B. Liu, B. An
}

Department of Neurology, Integrated Traditional Chinese and Western Medicine Hospital of Tongji, Medical College of Huazhong University of Science and Technology, Wuhan, China

Objective: To study the correlation factors influencing the length of stay (LOS) of cerebral infarction (CI) patients in Wuhan. Method: The clinical data of 1,403 patients diagnosed as CI by CT or MRI scans from December 2002 to February 2007 were retrospectively reviewed. All the patients had been divided into three groups according to their LOS (5 10 days, $11 \sim 14$ days and $15 \sim 20$ days). Influencing factors of each group such as gender, conscious state, diabetes, heart disease, stroke history, hereditary factors, Chinese traditional medicine treatments and neurological rehabilitation were assessed by Kruskal-Wallis nonparametric test analysis. The correlation factors were analyzed by logistic regression analysis. Result: There were significant differences in gender $\left(\mathrm{X}^{2}=3.9515, \mathrm{p}=0.0468\right)$, diabetes $\left(\mathrm{X}^{2}=6.1321\right.$, $\mathrm{p}=0.0133)$, conscious state $\left(\mathrm{X}^{2}=7.5728, \mathrm{p}=0.0059\right)$ and neurological rehabilitation $\left(X^{2}=15.9679, \mathrm{p}<000.1\right)$ among each group. Other factors such as heart disease, stroke history, hereditary history and Chinese traditional medicine treatments also had effects on the LOS, however, not obviously. Diabetes and neurological rehabilitation factors have significant relationships with LOS by logistic regression analysis. Their OR value were 1.634 and 1.701 respectively. Conclusion: The LOS of CI patients have the relationships with patients' gender, conscious state, diabetes as well as neurological rehabilitation treatments. Moreover, diabetes and neurological rehabilitation treatments were probably the most important factors for LOS of CI patients in Wuhan.

\section{Benefit of an Extended Stroke Unit to Acute Cerebral Infarction}

\section{O. Su, T. Lin, Y. Wu, Y. Ou, M. Cai, Z. Chen \\ Affiliated Hospital of Hainan Medical College, Haikou, China}

Objective: To evaluate the benefit of an extended stroke unit to acute cerebral infarction. Methods: Totally 102 patients with acute cerebral infarction in one week, who were chosen in the conditions: 
the length of the hospital stay Barthel Index (BI) was $30 \sim 60$ and the length of National Institutes of Health Stroke Scale (NIHSS) was $10 \sim 20.58$ Patients were randomized into extended stroke unit (ESU) and 44 patients were randomized into general ward (GW) to receive treatments for 3 weeks. Results: Compared with GW, the discharge of NIHSS and BI in ESU $(p<0.05)$ was more high in ESU. There was significant difference for NIHSS and BI in the groups of ESU after treatment for 3 weeks $(p<0.05)$, but was not significant difference for BI in the groups of GW. Conclusion: Extended stroke unit can decrease clinical neurological deficit and increase especially activities of daily life in acute cerebral infarction.

\section{Outcome and Safety Assessment of YIN Pattern of Acute Ischemic Stroke Treated with an Herbal Medicine Protocol}

\author{
Y. Huang, J. Pei, X. Wang
}

The First Department of Neurology, Guangdong Provincial

Hospital of Traditional Chinese Medicine, Guangzhou, China

Methods: A multi-center, single blinded, random and control clinical trial was adopted with 537 patients with AIS, they were divided into the treatment group (274 cases, Group A) treated with herbal protocol, and the control group (263 cases, Group B) treated with western medicine plus placebo, and AIS related indexes were assessed after treatment. Results: The baseline characters of are comparable Between Group A and Group B ( $p>0.05)$. 5 cases died in Group A and 7 cases died in Group B, which had no significant difference, $(p>0.05)$; Compared with the group $B$, the neurological deficit score (National Institute of Health Stroke Scale, NIHSS) determined on the 21 st day after treatment was ameliorated in the treatment group $(\mathrm{p}<0.05)$. Moreover, the 90-day follow-up showed that the severe disability rate was lower (BI $<75,20 \%$ vs. $26.3 \%$ ) $(\mathrm{p}<0.01)$, the complete rehabilitation and mild disability rate (mRS $0-1,59.3 \%$ vs. $67.9 \%$ ) were higher in the Group A than those in the Group B $(\mathrm{p}<0.05) .76$ cases of adverse reaction/events happened; which manifested as digestive symptom, skin action, bleeding tendency and aminopherase higher, but had no statistical difference in two groups. Conclusion: The HMP used in this study may improve neural function of patients in 21 days, and reduce the severe disability rate in those after 90 days treatment, with little adverse reaction with HMP.

\section{Effects of Leukoaraiosis on Outcomes of Mild Cognitive Impairment \\ L. Zheng, G. Xu, G. Lu, X. Liu \\ Department of Radiology, Nanjing General Hospital of PLA, Nanjing, China}

Objective: To evaluate the effects of co-exited leukoaraiosis (LA) on the outcomes of mild cognitive impairment (MCI). Methods: MCI were diagnosed with well-established criteria in in-patients and out-patients. Presence of LA was assessed with reference to results of magnetic resonance imaging (MRI) and computer tomography (CT). MCI patients were followed and their cognitive deteriorations were evaluated. Kaplan-Meier survival analysis was utilized for assessing the effects of LA on conversion of MCI to dementia. Results: Seventy-four patients with MCI were diagnosed based on clinical evaluation and neuropsychological assessment, 24 (32.4\%) of which had co-exited LA, and the other $50(67.6 \%)$ did not have LA. Fifteen of the $24(62.5 \%)$ MCI patients with LA developed dementia, while $9(18.0 \%)$ of $50 \mathrm{MCI}$ patients without LA developed dementia during 6-24 (13.2 \pm 6.8$)$ months follow-up. Kaplan-Meier analysis indicated there were more patients with LA developed dementia than patients without LA did $(\mathrm{p}>0.05)$. Conclusion: MCI patients with LA are more prone for progressing into dementia. These results suggest that early interventions and controlling the modifiable risk are warranted for dementia prevention in MCI patients with LA.

\section{The Application of 64-Slice CT Angiography in Evaluating Internal Vertebral Artery Stenosis}

X. Fan, X. Liu

Department of Neurology, Jinling Hospital, Nanjing

University School of Medicine, Nanjing, China

Objective: To evaluate the diagnostic accuracy of dual source 64-slice CT angiography for internal vertebral artery stenosis. Methods: Fifty-eight posterior circulation stroke or TIA patients with interpretable CTA and DSA of the vertebral arteries were selected from January 2007 to August 2007. In CTA, the grade of stenosis was determined using axial source, MIP, MPR as well as VR images. The images were blindly analyzed by 2 neuroradiologists using the NASCET criteria separately. Results: CTA agreed with DSA categorization of a stenosis in 102 of the 116 arteries. When the $70 \%$ stenosis was used as the cut-off value, the sensitivity, specificity, negative predictive value and the positive predicting value were $95 \%$, $97 \%, 0.89$ and 0.99 , respectively. Degree of artery stenosis on CTA closely correlated with that on DSA $(r=0.982, p<0.01)$. Conclusion: An excellent correlation and high accuracy were demonstrated between CTA and DSA on degree of stenosis, thus CTA appears to be an excellent screening test for vertebral artery stenosis.

\section{A Rare Variation of Abdominal Aorta Breaking in Two Found by Conventional Cerebral Angiography}

Y. Zhou, P. Shan, J. Xu

People's Liberation Arm 411 Hospital, Shanghai, China

Objective: To make clear a rare variation of abdominal aorta breaking in two and to investigate the cause of formation. Methods: Gadolinium-enhanced MRA with MIP and VRT procedure was used to find out the whole variation of abdominal aorta in an old Chinese female in whom the conventional angiography had failed to reveal this vascular abnormality. Results: MRA showed a clearly separated 
abdominal aorta. The upper part of abdominal aorta terminated under the two renal artery branches and lower part terminated at the level of $\mathrm{L}_{3-4}$. The compensated enlarged superior and inferior mesenteric artery connected these two isolated parts of abdominal aorta. Conclusion: This rare variation of abdominal aorta without any symptoms was probably caused by some external force during embryo.

\section{A Study of Relationship between Centrum Ovale Ischemic Lesions and Cerebral Atherosclerosis}

\author{
X. Fan, X. Liu, W. Zhu, G. Xu \\ Department of Neurology, Jinling Hospital, School of \\ Medicine, Nanjing University, Nanjing, China
}

Objective: To study the relationship between centrum ovale ischemic lesions and cerebral atherosclerosis, and to investigate the etiological mechanism of centrum ovale ischemic lesions. Methods: 46 patients with the centrum ovale ischemic lesions and 29 patients of controls were obtained from Nanjing Stroke Register database. Magnetic resonance imaging (MRI) and digital subtraction angiography were performed in all patients. Results: Compared with controls, prevalence of cerebral atherosclerosis and (or) stenosis in patients with centrum ovale ischemic lesions was higher $(p<0.05)$.The most frequent site of atherosclerosis and (or) stenosis was internal carotid artery, followed by vertebral artery, middle cerebral artery, posterior cerebral artery, anterior cerebral artery, common carotid artery and basilar artery. The prevalence of cerebral atherosclerosis and (or) stenosis in the elder patients was higher $(p<0.05)$. Conclusions: Cerebral atherosclerosis is a important pathogenesis of centrum ovale ischemic lesions.

\section{Clinical Analysis of 115 Cases of Cerebral Infarction on Youth Under 40}

\author{
C. Zheng, G. Xu, X. Liu \\ Department of Neurology, Wuwei People's Hospital, \\ Anhui Province, China
}

Objective: To evaluate the etiology, risk factors and clinical characters of youth cerebral infarction in the different age groups on this locality. Methods: To make a retrospective analysis according to the clinical material in 115 cases of cerebral infarction which were divided into juvenile groups (age ranged from 15 to 27 year-old) and young adults groups (age ranged from 28 to 40 year-old). Results: Among all cerebral infarction, juvenile groups had 29 cases; young adults groups had 86 cases. According to TOAST classification, there were 106 cases of cerebral infarction with clear cause. Among which Stroke of Other Determined Etiology were most frequent for juvenile, but the etiology was dispersed for young adults $(p<0.05)$. The important risk factors were male, hypertension, hyperlipidemia, smoking and alcohol abuse. Among which male and hypertension were obvious difference in the two groups $(p<0.01)$. The focus cerebral infarction in juvenile patients mostly occurred in anterior circulation and were usually found at least two, and the difference were significantly compared with young adults $(\mathrm{p}<0.05)$. The prognosis was good for the two groups, and the total effect treatment rate was $88.7 \%$. Conclusion: There was a different distribution in the juvenile groups and the young adults groups for the youth cerebral infarction, but most of them had clear causes. The prognoses are good if the etiology can be found and treated in the earlier period.

\section{Research Progress on Acute Cerebrovascular Diseases Complicated by Multiple Organ Dysfunction Syndromes}

\section{Z. Zhao}

Department of Neurology, Affiliated Changzheng Hospital of Second Military Medical University of PLA, Shanghai, China

Multiple organ dysfunction syndromes (MODS) is a progressive condition usually characterized by combined failure of two or several organs, along with all sorts of complications, usually after severe post-injury or postoperative for twenty-four hours. MODS is a dynamic and continuous process. MODS is one of severe complications of acute cerebrovascular disease (ACVD), incidence rate was $11-21 \%$ or so, usually lungs, heart, stomach intestine, kidney and metabolism system were involved in frequently. ACVD was combined failure of two or several organs; mortality was $41-87 \%$. MODS was one of fatal cause of patients with ACVD. The pathogenesis of ACVD complicated by MODS depended on acute reaction to stress, dysfunction of hypothalamus and brain stem, systemic inflammatory response syndrome (SIRS), and so on. Among them SIRS was fundamental. The pathogenesis had three pathways: (1) activated macrophage released cytokine and medium; (2) acute focal ischemiareperfusion and microcirculation disturbance; (3) endotoxemia. MODS proceed due to inflammatory reaction activated by etiological factor. This meant apoptosis and necrosis induced by SIRS. Apoptosis was critical in MODS and SIRS. The study of MODS focus on vascular endothelial cell (VEC).VEC protected adjacent tissue through priming and localization of focal inflammatory reaction. VEC was critical cell of MODS possibly. The prognosis was worse when ACVD complicated by MODS, so prevention and treatment grew more important. Neurological and relative organs' metergasis were monitored early and routinely. The recovery of brain's function was important measure to prevention and cure MODS. (1) active treatment of primary affection; (2) control of infection: emphasis on pulmonary infection, intestinal infection and urethral infection; (3) monitor metergasis of important organ, especially the disordered organ; (4) protect and support important organ, especially prevent acute renal failure (ARF). These measures could induce mortality of ACVD complicated by MODS. 


\section{Neuroprotective Effect of Intranasally Administrated Nerve Growth Factor in Patients with Acute Ischemic Stroke}

\author{
G. Xu, O. Yin, R. Zhang, X. Fan, X. Fan, X. Liu \\ Department of Neurology, Jinling Hospital, Nanjing \\ University School of Medicine, Nanjing, China
}

Stroke is a common and catastrophic disease which affects individuals with advanced ages. With the aging of population, prevalence of stroke is constantly increasing in recent years, especially in developing countries such as China. Ischemic stroke represent a large proportion of stroke in most regions all around world. The management of acute ischemic stroke requires an individually tailored approach that depended on the time the patients available for treatment initiation, the type and subtype of stroke, the severity of stroke, presence or absence of risk factors, resources available in the local clinical and many other factors.

Based on the hemi-ischemic theory, endangered neurons in the penumbra may be rescued if some neuroprotective interventions initiated promptly. The search for clinically effective neuroprotective drugs to rescue endangered brain tissue and prevent secondary brain damage after stroke has been disappointing. In spite of many neuroprotective agents showing efficacy after ischemic stroke in animals, none have produced significant neuronal protection when tested in clinical trials. The initial damage after brain ischemia is produced in the center of the lesion, defined as the ischemic core, where cells die within the first minutes after blood flow interruption. The ischemic penumbra is defined as an area of cerebral tissue surrounding the core. This area has a reduced perfusion and is amenable to be rescue by pharmacologic agents acting on some damage spreading mechanisms, such as increased extra-cellular glutamate concentrations, increased intracellular $\mathrm{Ca}^{2+}$, free radicals overproduction and exacerbated inflammatory response. Currently, neuroprotective strategies have focused on acting on only one of the mechanisms above described. However, some efforts have been recently made, to combine agents or interventions to increase the probability of success in this setting. Optimally, the use of a single pharmacologic agent or procedure interfering simultaneously in blocking two or more mechanisms of damage is highly desirable.

Nerve growth factor (NGF) is a good candidate to produce neuroprotective effects by inhibiting both glutamate-mediated excitotoxicity and inflammatory response, after ischemic insult to the brain. NGF was discovered more than 70 years ago via its action on explants of sensory and sympathetic neurons. As its name implies, it was identified as a factor that stimulated growth of processes and was thus considered to be an important agent during development and in adulthood. Identification of the trkA and p75 receptors for NGF has facilitated investigation of functional effects of the NGF system as well as its molecular mode of action. A more detailed functional view of NGF actions in vivo has been advanced by studies utilizing such powerful tools as transgenic animals and inhibition/sequestration or application of NGF. In vitro work has been advanced by selective agonists or antagonists for receptors or intracellular signaling pathways. One of the most important findings concerning the functional action of NGF has been its close association with the nociceptive system specifically, neural crest-derived nociceptors. NGF has become widely known as a trophic factor necessary to promote survival of nociceptors during the period of cell death late in development, including those that will subsequently replace expression of neurotrophin receptors with c-RET, the kinase subunit of the receptor complex for glial-derived neurotrophic factor. Removing NGF during this period results in a selective loss of nociceptive afferents and subsequent hypoalgesia. Studies with anti-NGF have revealed that $\mathrm{C}$-fiber nociceptors are also affected in that they display reduced sensitivity to noxious heat.

The neuroprotective effects of NGF have been recently shown in several experimental studies of acute and permanent middle cerebral artery occlusion in rodents. Pharmacokinetics of NGF and its pattern of adverse reactions are well-known, as the drug is used for treatment of Alzheimer Disease and pressure ulcer. As show by several in vivo studies, NGF is relatively safe and free of adverse reactions when administered in a single dose in focal or by oral administration.

Because NGF has a relatively larger molecular, it has a very low transmissivity through brain blood barrier (BBB) which will filtrate most organic molecular larger than $500 \mathrm{D}$. The bioavailability of NGF in brain tissue is very limited after intravenous administration. In a recent animal study, we have showed that NGF can bypass BBB, reach different brain areas and produce its biological effects after intranasal administration in rats. So in this study, we investigated the effectiveness of intranasal administrated NGF in treating acute ischemic stroke.

NGF has been demonstrated to be fast-acting, efficacious, and well tolerated in the acute treatment of stroke in rodents with comparable pharmacokinetic profiles for rats and mice. Therefore, the objective of the present study was to evaluate the efficacy and tolerability of intranasally administrated NGF versus placebo in the treatment of acute ischemic stroke. An expert panel of pediatric neurologists, including the study centers performing this study, recommended that a novel study design be adopted to address the anticipated high placebo response rates among stroke patients.

We conducted a double-blind, placebo-controlled, pilot clinical trial, recruiting patients from January 2004 to January 2006 in Jinling hospital which is attached to Nanjing University. Ethical approval was obtained from our Institutional Review Board before the study. Thirtysix patients with acute ischemic stroke with a National Institutes of Health Stroke Scale (NIHSS) score equal to or greater than 4. In all cases, CT scan was performed before enrollment to excluded hemorrhagic stroke. In all cases, ischemic stroke was confirmed with a second CT or MRI study within the first 24 hours of admission. Patients were randomized to receive either placebo or NGF nasal drop. Exclusion criteria included concurrent severe cardiovascular diseases, such as cardiac infarct within the previous 8 weeks, ventricular arrhythmia, second or third grade sinus-ventricular blockade or prolonged QT, and a therapeutic window over 24 hours after the first stroke symptom, disorders in coagulation system, concomitant malignant tumor, history of allergy to NGF, significant otorhinolaryngologic diseases, lung diseases. The use of anti-coagulants or anti-platelets was allowed, as patients received the optimal treatment protocol of ischemic stroke in our hospital. All patients or their closest family member gave written informed consent before enrolled in the study.

Once admitted to the study, patients were randomly allocated into placebo or NGF nasal drop arms. Patients treated with NGF nasal drop were administered with $1 \mathrm{mg}$ NGF dissolved in $1 \mathrm{ml}$ sodium succinate buffer at $\mathrm{pH}$ 6.0. Nasal drop were given to patients with prostration position over a 30-minute period. Alternating drops were practiced every 3 minutes between the left and right nares. Mouth and opposite naris were closed during administration so that the drops could be naturally inhaled deep into the nasal cavity. The same dosage 
and procedure were repeated for 7 days after the initial administration. One ml sodium succinate buffer without NGF as placebo were administrated to control group by the similar process for consecutive 7 days. After each nasal administration, patients were kept at prostration position for at least 4 hours.

Appearance, packaging and administration of placebo and NGF nasal drop were identical. The technique feasibility and pharmacological stability of NGF via nasal administration has been tested in rates by us and the results published elsewhere. The dosage of $1 \mathrm{mg}$ applied in this study was chosen with reference to that of topic administration of NGF in treating pressure ulcer. Also, we took advantage of the relatively long half life period of exogenous NGF in brain tissue to give a single dose of the drug per day during the treatment period.

Patients were followed up using the following clinical scales: NIHSS, applied on days 0, 7, 14 and 30; modified Rankin scale and Barthel index at day 30. All scales were applied by a single neurologist, previously trained to perform the measurements and blind to the treatment groups. Any suspected adverse reactions were recorded, along the first 7 days of follow-up. Laboratory values, the presence of comorbidity, the time after the first symptoms and the time from first symptoms until medication, were all recorded at admission. Sample size was not calculated before the start of the study, as this is a pilot trial to show the feasibility and safety of using the drug as neuroprotective in stroke patients.

Data were analysed blindly by a statistician, using the SPSS software version 11.0. Bi-variate comparisons were performed using independent t-test or Fisher exact test, for continuous and categorical variables respectively. ANOVA for repeated-measures was utilized to detect significant differences between the two treatment groups using NIHSS as dependent variable along days $0,7,14$ and 30. Modified Rankin scale at day 30 and Barthel index at day 30 were used to calculate relative risks (RRs), taking placebo group as a reference. The cut points were $0-2$ as good outcome for modified Rankin scale and 17 or more as good outcome for Barthel index.

All included patients were followed up until day 30, with the exception of two patients who died, one in NGF group and the other in control group. Eighteen patients were treated with NGF nasal drop and the other 18 with placebo. There were 23 males $(63.9 \%)$ and 13 females $(36.1 \%)$ in the study subjects. The age of the subjects ranged from 54 to $75(64.1 \pm 7.4$, median $=63)$ years. None of the variables concerning the demographic and risk factors for cerebrovascular diseases at admission differed significantly between the control and the NGF groups, which indicated that the two groups are comparable. Patients in NGF group started intranasal administration within
$6.5 \pm 3.4$ hours after the first symptom; while patients control group started intranasal administration with $6.1 \pm 3.5$ hours after the initial symptom. There were no significant differences concerning the time of intranasal administration $(\mathrm{p}=0.65)$.

One patient from the NGF nasal drop group during the follow-up, owing to an event independent of NGF nasal drop treatment (the patient suffered a myocardial infarction). Another patient from control group died from heart failure. NIHSS at admission was $4.8 \pm 0.8$ for NGF group and $4.7 \pm 0.9$ for control group. No significant differences were detected concerning the baseline NIHSS scores $(p=0.70)$. NIHSS was found normally distributed in both groups at admission. In order to compare the NIHSS throughout days 0 to 30 between treatment groups, ANOVA for repeated-measures was used. That analysis takes into account for any possible difference in NIHSS at admission. It was detected that NGF group was statistically different from placebo group ( $3.2 \pm 0.7$ vs. $4.2 \pm 0.6, p<0.05)$. To assess the time dependency of the neuroprotective effect of NGF nasal drop, clinical improvement was calculated as the subtraction of NIHSS at admission minus the value of NIHSS at day 30 for each patient. The results showed the clinical improvement was super in NGF group than in controls $(-1.6 \pm 0.5$ vs. $-0.5 \pm 0.6, p<0.05)$. Using the cutting points defined in methods, the modified Rankin scale at day 30 gave an estimate risk of $\mathrm{RR}=0.201(95 \% \mathrm{CI}: 0.06$ and 0.77$)$ for NGF nasal drop group, while the Barthel index was also significantly improved ( $\mathrm{p}<0.05$, exact Fisher's test).

No adverse reactions related to the NGF administration were identified during treatment and follow-ups. Severity of the brain infarction and age of the patients are well-known factors affecting the outcome after stroke. Other variables related to the prognosis include serum glucose levels, blood pressure and serum cholesterol levels at admission. As a result of randomization, all of these parameters were homogeneous between the NGF and placebo groups of this pilot trial. Regarding the neuroprotective mechanisms of NGF, it is known that the drug is able to prevent inflammatory-mediated damages and NMDA-mediated neurotoxicity. As enhanced glutamate release is involved in the damage after ischemia, NGF nasal drop is perhaps acting at this point to protect tissue from further damage. The wellknown anti-inflammatory actions of NGF may also contribute to prevention of the damage in the acute phase of ischemia.

As excessive inflammatory response has been involved as one of the mechanisms spreading the damage from the core to the penumbra region, NGF may help to attenuate this deleterious process. Results from the present study are promising and support further research to define the role of NGF administrated intranasally as a neuroprotective strategy in patients with acute stroke. 